\title{
New FOCUS results on charm mixing and CP violation
}

\author{
Stefano Bianco * \\ Laboratori Nazionali di Frascati dell'INFN - via E. Fermi 40, Frascati I-00044
}

\begin{abstract}
We present a summary of recent results on CP violation and mixing in the charm quark sector based on a highstatistics sample collected by photoproduction experiment FOCUS (E831 at Fermilab). We have measured the difference in lifetimes for the $D^{0}$ decays: $D^{0} \rightarrow K^{-} \pi^{+}$and $D^{0} \rightarrow K^{-} K^{+}$. This translates into a measurement of the $y_{C P}$ mixing parameter in the $D^{0} \bar{D}^{0}$ system, under the assumptions that $K^{-} K^{+}$is an equal mixture of CP odd and CP even eigenstates, and CP violation is negligible in the neutral charm meson system. We verified the latter assumption by searching for CP violating asymmetry in the Cabibbo suppressed decay modes $D^{+} \rightarrow K^{-} K^{+} \pi^{+}, D^{0} \rightarrow K^{-} K^{+}$and $D^{0} \rightarrow \pi^{-} \pi^{+}$. We report preliminary results on a measurement of the branching ratio $\Gamma\left(D^{*+} \rightarrow \pi^{+}\left(K^{+} \pi^{-}\right)\right) / \Gamma\left(D^{*+} \rightarrow \pi^{+}\left(K^{-} \pi^{+}\right)\right)$.
\end{abstract}

\section{INTRODUCTION}

Particle-antiparticle mixing in the charm sector has distinctive features that make it a high sensitivity probe to search for New Physics. Re-

\footnotetext{
*on behalf of the FOCUS Collaboration. Coauthors are: J.M. Link, V.S. Paolone, M. Reyes, P.M. Yager (UC DAVIS); J.C. Anjos, I. Bediaga, C. Göbel, J. Magnin, J.M. de Miranda, I.M. Pepe, A.C. dos Reis, F. Simão (CPBF, Rio de Janeiro); S. Carrillo, E. Casimiro, H. Mendez, A.Sánchez-Hernández,, C. Uribe, F. Vasquez (CINVESTAV, México City); L. Cinquini, J.P. Cumalat, J.E. Ramirez, B. O'Reilly, E.W. Vaandering (CU Boulder); J.N. Butler, H.W.K. Cheung, I. Gaines, P.H. Garbincius, L.A. Garren, E. Gottschalk, S.A. Gourlay, P.H. Kasper, A.E. Kreymer, R. Kutschke (Fermilab); S. Bianco, F.L. Fabbri, S. Sarwar, A. Zallo (INFN Frascati); C. Cawlfield, D.Y. Kim, K.S. Park, A. Rahimi, J. Wiss (UI Champaign); R. Gardner (Indiana ); Y.S. Chung, J.S. Kang, B.R. Ko, J.W. Kwak, K.B. Lee, S.S. Myung, H. Park (Korea University, Seoul); G. Alimonti, M. Boschini, D. Brambilla, B. Caccianiga, A. Calandrino, P. D'Angelo, M. DiCorato, P. Dini, M. Giammarchi, P. Inzani, F. Leveraro, S. Malvezzi, D. Menasce, M. Mezzadri, L. Milazzo, L. Moroni, D. Pedrini, F. Prelz, M. Rovere, A. Sala, S. Sala (INFN and Milano); T.F. Davenport III (UNC Asheville); V. Arena, G. Boca, G. Bonomi, G. Gianini, G. Liguori, M. Merlo, D. Pantea, S.P. Ratti, C. Riccardi, P. Torre, L. Viola, P. Vitulo (INFN and Pavia); H. Hernandez, A.M. Lopez, L. Mendez, A. Mirles, E. Montiel, D. Olaya, J. Quinones, C. Rivera, Y. Zhang (Mayaguez, Puerto Rico); N. Copty, M. Purohit, J.R. Wilson (USC Columbia); K. Cho, T. Handler (UT Knoxville); D. Engh, W.E. Johns, M. Hosack, M.S. Nehring, M. Sales, P.D. Sheldon, K. Stenson, M.S. Webster (Vanderbilt); M. Sheaff (Wisconsin, Madison); Y. Kwon (Yonsei University, Korea).
}

cently, the possibility of collecting large, highquality samples of fully reconstructed $D$ meson decays have germinated several new results [1]. In $\S 2$ we review the basic formalism of charm mixing and $\mathrm{CP}$ violation (CPV), as well as the experimental techniques for measuring the mixing parameters. After briefly describing the FOCUS detector in $\S 3$, in $\S 4$ we report on a new measurement of the difference in lifetimes for the $D^{0}$ decays: $D^{0} \rightarrow K^{-} \pi^{+}$and $D^{0} \rightarrow K^{-} K^{+}$. In $\S 5$ we show how we searched for CPV asymmetry in the Cabibbo suppressed decay modes $D^{+} \rightarrow K^{-} K^{+} \pi^{+}, D^{0} \rightarrow K^{-} K^{+}$and $D^{0} \rightarrow$ $\pi^{-} \pi^{+}$. Finally, in $\S 6$ we report preliminary results on a measurement of the branching ratio $\Gamma\left(D^{*+} \rightarrow \pi^{+}\left(K^{+} \pi^{-}\right)\right) / \Gamma\left(D^{*+} \rightarrow \pi^{+}\left(K^{-} \pi^{+}\right)\right)$.

\section{CHARM MIXING AND CPV}

Let us recall the key features of particleantiparticle mixing [2] [3] 近. Because of weak interactions, flavor $f=s, c, b$ of a generic pseudoscalar neutral meson $P^{0}$ is not conserved. Therefore, it will try and decay with new mass eigenstates $P_{1,2}^{0}$ which no longer carry definite flavor $f$ : they are new states with different mass and lifetime $\left|P_{1,2}^{0}\right\rangle \propto\left(p\left|P^{0}\right\rangle \pm q\left|\bar{P}^{0}\right\rangle\right)$ where complex parameters $p$ and $q$ account for any CPV. The time evolution of $\left|P^{0}(t)\right\rangle$ is given by the Schrödinger equation. After a time $t$ the probability of finding the state $P^{0}$ transformed into 


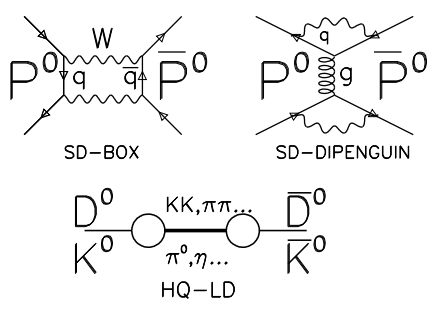

Figure 1. Box (proportional to $\left(m_{q}^{2}-m_{u}^{2}\right) / m_{W}^{2}$ ), penguin, and long-distance diagrams for mixing.

$$
\begin{aligned}
& \bar{P}^{0} \text { is } \\
& \begin{aligned}
\left|\left\langle\bar{P}^{0} \mid P^{0}(t)\right\rangle\right|^{2} \propto & \left|\frac{q}{p}\right|^{2} e^{-\Gamma_{1} t}\left[1+e^{\Delta \Gamma t}+\right. \\
& \left.2 e^{\frac{\Delta \Gamma}{2} t} \cos (\Delta m t)\right]
\end{aligned}
\end{aligned}
$$

with definitions $\Delta m \equiv m_{1}-m_{2}, \Delta \Gamma=\Gamma_{1}-\Gamma_{2}$ and $\bar{\Gamma} \equiv\left(\Gamma_{1}+\Gamma_{2}\right) / 2$. The two states will oscillate with a rate expressed by $\Delta m$ and $\Delta \Gamma$, which are usually calibrated by the average decay rate by means of the mixing parameters

$x \equiv \Delta m / \bar{\Gamma} \quad y \equiv \Delta \Gamma /(2 \bar{\Gamma})$

In the case of charm mesons, because of the Cabibbo-favored decay mechanism and the large phase space available for their decay, decay widths are very similar $(y \ll 1)$, and the timeintegrated ratio of mixed and nonmixed rates is

$r \equiv \frac{\Gamma\left(D^{0} \rightarrow \bar{D}^{0} \rightarrow \bar{f}\right)}{\Gamma\left(D^{0} \rightarrow f\right)}=\left|\frac{q}{p}\right|^{2} \frac{x^{2}+y^{2}}{2}$

Theoretical estimates of $x$ traditionally fall into two main categories, short distance (SD) and heavy quark/long distance (HQ-LD): the former arise from the box diagram (Fig. 1]a), with GIM mechanism suppressing the charm case (Tab.11) or the dipenguin diagram, the latter come from QCD diagrams and final state interactions (FSI) such as rescattering of quarks with known intermediate light states (Fig.11c). Recently, OPEbased approaches have also been proposed[6].

An important comment was made recently [7] on the possibility of measuring $y$ separately from $x$. Indeed, $x \neq 0$ means that mixing is genuinely produced by $D^{0} \bar{D}^{0}$ transitions (either SD
Table 1

Box diagram contributions to mixing, and compilation of mixing parameters (95\% CL ) [5].

\begin{tabular}{lccrr}
\hline$P^{0}$ & $q$ & $S D / L D$ & $x$ & $y$ \\
\hline$K^{0}(d \bar{s})$ & $\mathrm{c}$ & $S D \sim L D$ & 0.474 & 0.9965 \\
$D^{0}(c \bar{u})$ & $\mathrm{s}$ & $S D \ll L D$ & $<0.03$ & $-0.06<y<0.01$ \\
$B_{d}^{0}(d \bar{b})$ & $\mathrm{t}$ & $S D \gg L D$ & 0.73 & $?$ \\
$B_{s}^{0}(s \bar{b})$ & $\mathrm{t}$ & $S D \gg L D$ & $>15.7$ & $<0.16$ \\
\hline
\end{tabular}

or HQ-LD, or both), while $y \neq 0$ means that the fast-decaying component $D_{1}^{0}$ quickly disappears, leaving the slow-decaying component $D_{2}^{0}$ behind, which is a mixture of $D^{0}$ and $\bar{D}^{0}$. Infinite discussion is active on the extent to which the three contributions are dominant: consensus seems to exist on the HQ-LD being, in the case of charm mesons, larger than the SD, and in any case small. Standard Model predictions are

$x, y<10^{-7}-10^{-3} \quad r^{S M}<10^{-10}-10^{-4}$

still somewhat below the PDG2000 limit [5] $r<$ $4.1 \times 10^{-4}$. Any observation of $D^{0} \bar{D}^{0}$ mixing above the predicted level, once HQ-LD effects are understood, is a signal that new physics contributions are adding to the box diagrams. A recent compilation of predictions on $D^{0} \bar{D}^{0}$ mixing is in Ref. [8]. Traditionally, $D^{0} \bar{D}^{0}$ mixing is searched for by means of event-counting techniques, while advances in event statistics now allow studies of the $y$ parameter.

\subsection{Wrong sign vs right sign counting}

Mixing is searched for in the decay chains

$$
\begin{aligned}
D^{*+} & \rightarrow D^{0} \pi^{+} \\
D^{0} & \Rightarrow \bar{D}^{0} \\
\bar{D}^{0} & \rightarrow K^{+} \pi^{-}, K^{+} \pi^{-} \pi^{+} \pi^{-}, K^{+} \ell^{-} \bar{\nu}_{\ell}
\end{aligned}
$$

with the flavor of the neutral $D$ meson at production and at decay given by the sign of $\pi^{+}$and $K^{-}$respectively. In the case of a hadronic final state, life is complicated by pollution of the mixing by the Doubly-Cabibbo-Suppressed Decay $D^{0} \rightarrow K^{+} \pi^{-}$, proportional to $\tan ^{4} \theta_{C}$. The measurable $r_{W S}$ - the rate of wrong-sign events - has therefore contributions [9] from DCSD, in- 
terference, and mixing

$$
\begin{aligned}
r_{W S} & =\Gamma\left(D^{0} \rightarrow f\right) / \Gamma\left(\bar{D}^{0} \rightarrow f\right) \\
& =\frac{e^{-\bar{\Gamma} t}}{4}\left|\left\langle f|H| D^{0}\right\rangle\right|_{C F}^{2}\left|\frac{q}{p}\right|^{2}\left(X+Y t+Z t^{2}\right) \\
X & \equiv 4|\lambda|^{2} \\
Y & \equiv 2 \Re(\lambda) \Delta \Gamma+4 \Im(\lambda) \Delta m \\
Z & \equiv(\Delta m)^{2}+(\Delta \Gamma)^{2} / 4 \\
\lambda & \equiv \frac{p}{q} \frac{\left\langle f|H| D^{0}\right\rangle_{D C S}}{\left\langle f|H| \bar{D}^{0}\right\rangle_{C F}}
\end{aligned}
$$

The $\mathrm{X}$ term (pure DCS) is characterized by exponential decay time behavior, unlike the $\mathrm{Z}$ term (pure mixing), and this feature can in principle be used to suppress the DCS pollution. The Y (interference) term receives contributions from $\Im(\lambda)$, which can be nonzero if a) CPV is present, thus introducing a phase $\varphi \sim \arg \left(V_{c d} V_{u d}^{*} / V_{c s} V_{u s}^{*}\right)$ in $p / q$; and/or b) a strong phase $\delta$ is present, due to different FSI in the DCS and mixed CF decays. By assuming CP conservation, i.e., $|p / q|=1$, defining

$e^{i \varphi} \equiv \frac{p}{q} \quad e^{i \delta} \sqrt{r_{D C S}} \equiv \frac{\left\langle f|H| D^{0}\right\rangle_{D C S}}{\left\langle f|H| \bar{D}^{0}\right\rangle_{C F}}$

and measuring $t$ in units of $\bar{\Gamma}$ we can write a simpler expression for $r_{W S}$

$r_{W S} \propto e^{-t}\left[r_{D C S}+t^{2}(r / 2)+t \sqrt{2 r r_{D C S}} \cos \phi\right](8)$ where the interference angle is given by $\phi=$ $\arg (i x+y)-\varphi-\delta$. Equation 8 shows how a meaningful quote of the $r$ result must specify which assumptions where made on the CPV and strong angles $\varphi$ and $\delta$. In particular, it was recently pointed out in 10]11 how the possibility of a nonzero strong phase $\delta$, which vanishes in the limit of unbroken $\mathrm{SU}(3)$ symmetry, should in fact be carefully taken into account in $D^{0} \bar{D}^{0}$ mixing, due to the experimentally known feature of $\mathrm{SU}(3)$ to be badly broken in $\mathrm{D}$ decays. If one assumes CP invariance $(\varphi=0)$, then

$$
\begin{gathered}
r_{W S} \propto e^{-t}\left\{r_{D C S}+(r / 2) t^{2}+\left(y^{\prime} \sqrt{r_{D C S}}\right) t\right\} \\
y^{\prime} \equiv y \cos \delta-x \sin \delta \quad x^{\prime} \equiv x \cos \delta+y \sin \delta
\end{gathered}
$$

The alternative option in counting techniques is the use of semileptonic final states $K \ell \nu$, which do not suffer from DCSD pollution but are harder experimentally.

\subsection{Lifetime difference measurements}

The $y$ parameter can be determined directly by measuring the lifetimes of $\mathrm{CP}=+1$ and $\mathrm{CP}=-1 \mathrm{fi}-$ nal states - such as $K^{+} K^{-}$and $\pi^{+} \pi^{-}(\mathrm{CP}=+1)$, $K_{S} \phi(\mathrm{CP}=-1)-$ and $K^{-} \pi^{+}(\operatorname{mixed} \mathrm{CP})$, assuming both $\mathrm{CP}$ conservation, i.e., that $D_{1}^{0}$ and $D_{2}^{0}$ are indeed $\mathrm{CP}$ eigenstates, and that $K \pi$ is a mixed-CP eigenstate. We shall call such parameter $y_{C P}$, and if the above assumptions are verified

$y=y_{C P}=\frac{\tau(D \rightarrow K \pi)}{\tau(D \rightarrow K K)}-1$

In principle, a measurement of $y$ would allow, along with an independent measurement of $r$, limits to be set on $x$.

\subsection{CPV asymmetries}

The assumption of negligible CPV in the charm system is important in the study of mixing. CPV occurs if the decay rate for a particle differs from the decay rate of its CP-conjugate particle [2]. In charm meson decays (as well as in $K$ and $B$ decays), two classes of $\mathrm{CP}$ violation exist: indirect and direct. In neutral charm meson decays, indirect $\mathrm{CPV}$ may arise due to $D^{0} \bar{D}^{0}$ mixing. In the case of direct violation, $\mathrm{CP}$ violating effects occur in a decay process only if the decay amplitude is the sum of two different parts, whose phases are made of a weak (CKM) and a strong contribution due to final state interactions [12]

$A \equiv a e^{i \delta_{1}}+b e^{i \delta 2}$

The weak contributions to the phases change sign when going to the $\mathrm{CP}$-conjugate process, while the strong ones do not. In singly Cabibbosuppressed $D$ decays, penguin terms in the effective Hamiltonian may provide the different phases of the two weak amplitudes. The $\mathrm{CP}$ asymmetry will then be

$$
\begin{aligned}
A_{C P} & \equiv \frac{|A|^{2}-|\bar{A}|^{2}}{|A|^{2}+|\bar{A}|^{2}} \\
& =\frac{2 \Im\left(a b^{*}\right) \sin \left(\delta_{2}-\delta_{1}\right)}{|a|^{2}+|b|^{2}+2 \Re\left(a b^{*}\right) \cos \left(\delta_{2}-\delta_{1}\right)}
\end{aligned}
$$

Compared to the strange and bottom sectors, the SM predictions of CPV for charm decays are much smaller, making the charm sector a good place to test the SM and to look for evidence 
Table 2

Measured CP asymmetries $\left(\times 10^{-2}\right)$. References to quoted results are in Ref. [14]

\begin{tabular}{llll}
\hline Experiment & $D^{+} \rightarrow K^{-} K^{+} \pi^{+}$ & $D^{0} \rightarrow K^{-} K^{+}$ & $D^{0} \rightarrow \pi^{-} \pi^{+}$ \\
\hline E687 & $-3.1 \pm 6.8$ & $+2.4 \pm 8.4$ & \\
CLEO II & & $+8.0 \pm 6.1$ & \\
E791 & $-1.4 \pm 2.9$ & $-1.0 \pm 4.9 \pm 1.2$ & $-4.9 \pm 7.8 \pm 3.0$ \\
FOCUS[14] & $+0.6 \pm 1.1 \pm 0.5$ & $-0.1 \pm 2.2 \pm 1.5$ & $+4.8 \pm 3.9 \pm 2.5$ \\
\hline
\end{tabular}

of new physics. In the $\mathrm{SM}$, direct $\mathrm{CP}$ violating asymmetries in $D$ decays are predicted to be largest in singly Cabibbo-suppressed decays, at most $10^{-3}$, and non-existent in Cabibbo-favored and doubly Cabibbo-suppressed decays 2]. However, a CP asymmetry could occur in the decay modes $D \rightarrow K_{s} \mathrm{n} \pi$ due to interference between Cabibbo-favored and doubly Cabibbo-suppressed decays.

\section{FOCUS}

The data for this paper were collected in the wideband photoproduction experiment FOCUS during the Fermilab 1996-1997 fixed-target run. FOCUS is a considerably upgraded version of a previous experiment, E687 13]. In FOCUS, a forward multi-particle spectrometer is used to measure the interactions of high-energy photons on a segmented $\mathrm{BeO}$ target. We obtained a sample of over 1 million fully reconstructed charm particles in the three major decay modes: $D^{0} \rightarrow$ $K^{-} \pi^{+}, K^{-} \pi^{+} \pi^{-} \pi^{+}$and $D^{+} \rightarrow K^{-} \pi^{+} \pi^{+}$.

The FOCUS detector is a large-aperture, fixedtarget spectrometer with excellent vertexing, particle identification, and reconstruction capabilities for photons and $\pi^{0}$ 's. A photon beam is derived from the bremsstrahlung of secondary electrons and positrons with an $\approx 300 \mathrm{GeV}$ endpoint energy produced from the $800 \mathrm{GeV} / c$ Tevatron proton beam. The charged particles that emerge from the target are tracked by two systems of silicon microvertex detectors. The upstream system, consisting of four planes (two views in two stations), is interleaved with the experimental target, while the other system lies downstream of the target and consists of twelve planes of microstrips arranged in three views. These detectors provide high-resolution separation of primary (production) and secondary (decay) vertices with an average proper time resolution of $\approx 30 \mathrm{fs}$ for two-track vertices. The momentum of a charged particle is determined by measuring its deflections in two analysis magnets of opposite polarity with five stations of multiwire proportional chambers. Three multicell threshold Cerenkov counters are used to discriminate between pions, kaons, and protons, and complement the electron identification provided by the em calorimetry. For each charged track, the Cerenkov particle identification algorithm generates a set of $\chi^{2}$-like variables $W_{i} \equiv-2 \log$ (likelihood) where $i$ ranges over the electron, pion, kaon, and proton hypotheses.

\section{SEARCH FOR CP-VIOLATING ASYMMETRIES}

We have studied 14 the Cabibbo-suppressed decay modes that have the largest combination of branching fraction and detection efficiency. For this reason we selected the all-charged decay modes $D^{+} \rightarrow K^{-} K^{+} \pi^{+}, D^{0} \rightarrow K^{-} K^{+}$, and $D^{0} \rightarrow \pi^{-} \pi^{+}$(charge conjugate state implied, unless otherwise noted). In $D$ decays the charged $D$ is self-tagging and the neutral $D$ is tagged as a $D^{0}$ or as a $\bar{D}^{0}$ by using the sign of the bachelor pion in the $D^{* \pm}$ decay. The CP asymmetry parameter measures the direct $\mathrm{CP}$ asymmetry in the case of $D^{+}$and the combined direct and indirect CP asymmetries for $D^{0}$.

Before searching for CP asymmetry we must account for differences, at the production level, between $D$ and $\bar{D}$ in photoproduction (the hadronization process, in the presence of remnant quarks from the nucleon, gives rise to production asymmetries 15]). This is done by normalizing to the Cabibbo-favored modes $D^{0} \rightarrow K^{-} \pi^{+}$and $D^{+} \rightarrow K^{-} \pi^{+} \pi^{+}$, with the additional benefit that most of the corrections due to inefficiencies cancel out, reducing systematic uncertainties. An 
implicit assumption is that there is no measurable CPV in the Cabibbo-favored decays. The $\mathrm{CP}$ asymmetry can be written as:

$$
A_{C P}=[\eta(D)-\eta(\bar{D})] /[\eta(D)+\eta(\bar{D})]
$$

where $\eta$ is (considering, for example, the decay mode $\left.D^{0} \rightarrow K^{-} K^{+}\right)$:

$\eta(D)=\frac{N\left(D^{0} \rightarrow K^{-} K^{+}\right)}{N\left(D^{0} \rightarrow K^{-} \pi^{+}\right)} \frac{\epsilon\left(D^{0} \rightarrow K^{-} \pi^{+}\right)}{\epsilon\left(D^{0} \rightarrow K^{-} K^{+}\right)}$

where $N\left(D^{0} \rightarrow K^{-} K^{+}\right)$is the number of reconstructed candidate decays and $\epsilon\left(D^{0} \rightarrow K^{-} K^{+}\right)$ is the efficiency obtained from montecarlo simulations.

Table 2 shows the asymmetry numbers obtained by FOCUS, compared to previous published asymmetry measurements. The statistical error of the neutral decay channel is not as good as the charged one, since $D^{*}$ tagging is necessary to determine the flavor of the parent $D^{0}$. There is no clear evidence for CPV in our measurements, which correspond to new limits two-three times better than the previous measurements by E791.

\section{LIFETIME DIFFERENCES AND $y_{C P}$ MIXING PARAMETER}

We have studied [16] the $y_{C P}$ mixing parameter by measuring the difference in lifetimes for the $D^{0}$ decays: $D^{0} \rightarrow K^{-} \pi^{+}$and $D^{0} \rightarrow K^{-} K^{+}$.

\subsection{Event selection}

The cuts used to obtain a clean signal were designed to produce a nearly flat efficiency in reduced proper time $t^{\prime} \equiv(\ell-N \sigma) /(\gamma \beta c)$, which is defined as the proper time subtracted eventby-event by the minimum amount of detachment required between primary and secondary vertices. Our quoted result was based on requiring a minimum $\sigma_{\ell}$ detachment and kaon hypothesis over pion hypothesis in Cerenkov response favored for kaon candidates. Then we either require a $D^{*}$ tag, or a set of more stringent cuts, such as more stringent Cerenkov requirements on kaons and pions, momenta of decay particles balancing each other, primary vertex inside the target material, and resolution of proper time less than $60 \mathrm{fs}$. The $D^{*}$ tagged sample has a better signal-to-noise ratio, while the inclusive sample accommodates larger sample size. From the combination of two samples, we obtain $119738 D \rightarrow K \pi$ and 10331 $D \rightarrow K K$ events (Fig. 2).
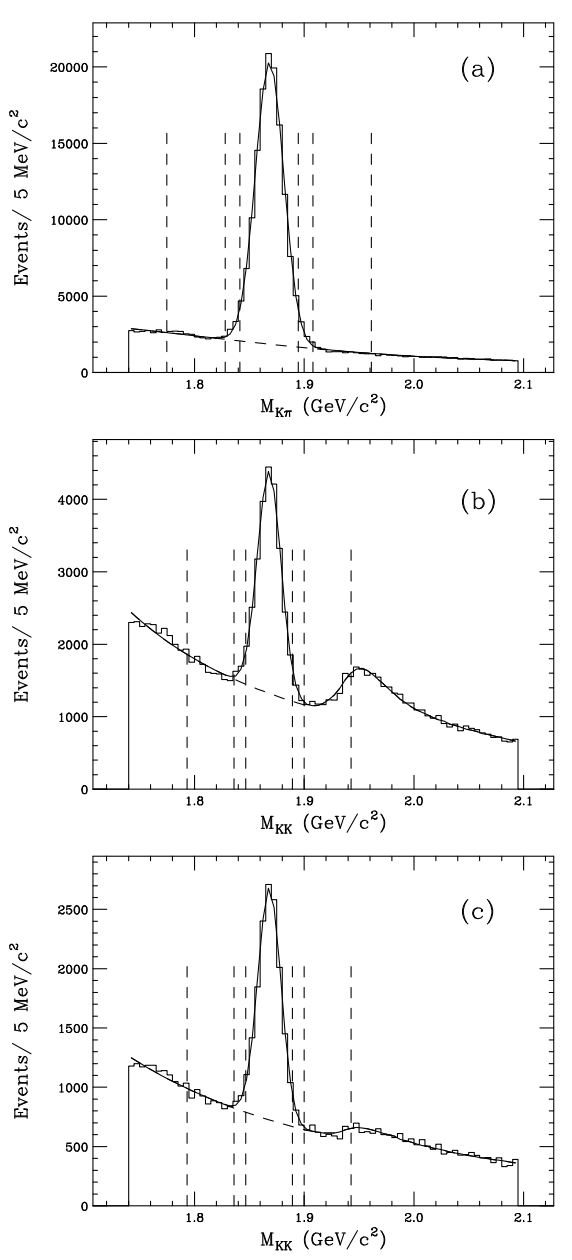

Figure 2. (a) Signal for $D^{0} \rightarrow K^{-} \pi^{+}$with a detachment cut of $\ell / \sigma>5$ and $W_{\pi}-W_{K}>4$. The yield is $119738 K^{-} \pi^{+}$signal events. (b,c) Signals for $D^{0} \rightarrow K^{-} K^{+}$with a detachment cut of $\ell / \sigma>5$. The reflection in the background at higher masses is due to contamination from misidentified $D^{0} \rightarrow K^{-} \pi^{+}$. (b) Requiring $W_{\pi}-W_{K}>1$, we obtain a yield of $16532 K^{-} K^{+}$ signal events. (c) Requiring $W_{\pi}-W_{K}>4$, we obtain a yield of $10331 \mathrm{~K}^{-} \mathrm{K}^{+}$signal events. The vertical dashed lines indicate the signal and sideband regions used for the lifetime and $y_{\mathrm{CP}}$ fits. 


\subsection{Fitting technique}

The $D^{0} \rightarrow K^{-} K^{+}$sample is characterized by a prominent reflection background coming from misidentified $D^{0} \rightarrow K^{-} \pi^{+}$decays (Fig. 2). We accommodate the reflection effect by using a modified version of the mass sideband subtraction fitting technique used in the E687 experiment 13. The amount of $D^{0} \rightarrow K^{-} \pi^{+}$reflection is obtained by a mass fit to the $K^{-} K^{+}$sample and the shape of the reflection is deduced from a highstatistics montecarlo sample. We assume that the time evolution of the reflection is described by the lifetime of $D^{0} \rightarrow K^{-} \pi^{+}$and we fit the reduced proper time distributions of the $D^{0} \rightarrow K^{-} \pi^{+}$ and $D^{0} \rightarrow K^{-} K^{+}$samples at the same time. The fit parameters are the $D \rightarrow K \pi$ lifetime, the lifetime asymmetry $y_{C P}$, and the number of background events under each $D^{0} \rightarrow K^{-} \pi^{+}$and $D^{0} \rightarrow K^{-} K^{+}$signal region. The signal contributions for the $D^{0} \rightarrow K^{-} \pi^{+}, D^{0} \rightarrow K^{-} K^{+}$and the reflection from the misidentified $D^{0} \rightarrow K^{-} \pi^{+}$in the reduced proper time histograms are described by a term

$f\left(t^{\prime}\right) \exp \left(-t^{\prime} / \tau\right)$

in the fit likelihood function. The function $f\left(t^{\prime}\right)$, determined from montecarlo, covers any deviation of the reduced proper time distribution from a pure exponential due to acceptance (Fig. 3).

The background yield parameters are either left floating, or fixed to the number of events in mass sidebands using a Poisson penalty term in the fit likelihood function. We choose $200 \mathrm{fs}$ as the bin size of the reduced proper time, which is large compared to our proper time resolution. Twenty bins are used in the fit (Fig. (1).

The systematic errors are estimated by changing the selection cuts and by trying different fitting methods. We tested the Cerenkov identification hypothesis for kaon candidates and the minimum detachment required between primary and secondary vertices. The former affects the level of reflection backgrounds, the latter affects the amount of non-charm backgrounds. We tried different numbers of histogram bins and two options of background handling, as stated in the previous subsection. Other variations of selection and fitting yielded results consistent with our number.

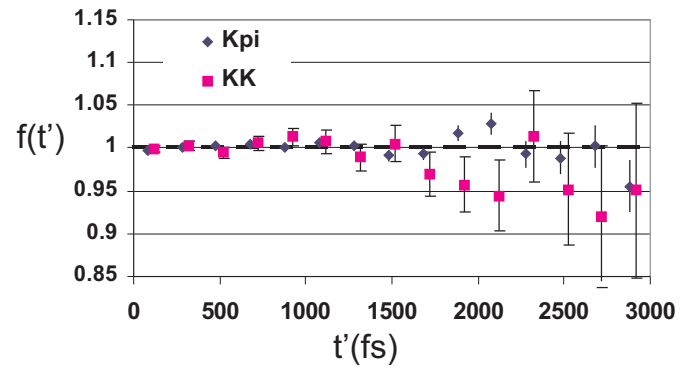

Figure 3. Montecarlo correction factors for $D^{0} \rightarrow K^{-} \pi^{+}$and $K^{-} K^{+}$for $\ell / \sigma>5$ and $W_{\pi}-W_{K}>4$. We have offset the $K^{-} K^{+}$points slightly for clarity and have given them "flats" on their error bars. Montecarlo corrections are rather slight with these cuts and the corrections for $D^{0} \rightarrow K^{-} \pi^{+}$are the same within errors as those for $D^{0} \rightarrow K^{-} K^{+}$.

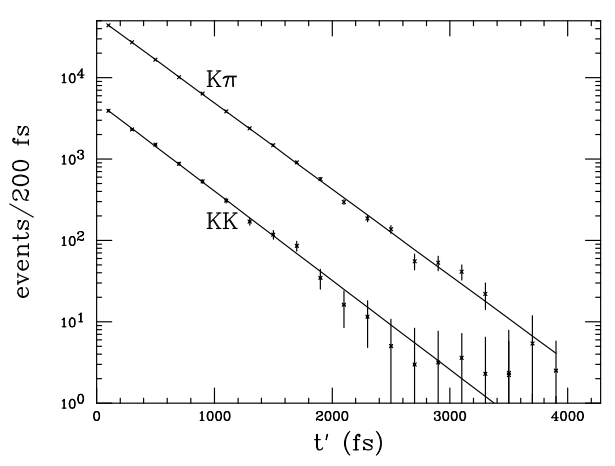

Figure 4. Signal versus reduced proper time for $D^{0} \rightarrow K^{-} \pi^{+}$and $K^{-} K^{+}$requiring $W_{\pi}-W_{K}>$ 4 and $\ell / \sigma>5$. The fit is over 20 bins of $200 \mathrm{fs}$ bin width. The data is background subtracted and includes the (very small) montecarlo correction.

We obtained

$$
\begin{array}{r}
y_{C P}=(3.42 \pm 1.39 \pm 0.74) \% \\
\tau(D \rightarrow K \pi)=409.2 \pm 1.3 \mathrm{fs}
\end{array}
$$

Our result on $\tau(D \rightarrow K \pi)$ has a statistical error only. Detailed systematics studies, including absolute distance scale, are needed to obtain the final number. 


\section{PRELIMINARY MEASUREMENT OF $\Gamma\left(D^{*+} \rightarrow \pi^{+}\left(K^{+} \pi^{-}\right)\right) / \Gamma\left(D^{*+} \rightarrow\right.$ $\left.\pi^{+}\left(K^{-} \pi^{+}\right)\right)$}

As described in $\S 2.1$, the $D^{0}$ can decay to $K^{+} \pi^{-}$through two physical processes: 1) by a DCS decay or 2) by mixing to $\bar{D}^{0}$ followed by the $\mathrm{CF}$ decay to $K^{+} \pi^{-}$. The Standard Model predicts a DCS to CF branching ratio $\left(r_{D C S}\right)$ of the order $\tan ^{4} \theta_{c} \simeq 0.25 \%$. The Standard Model predictions for $D^{0} \bar{D}^{0}$ mixing rate were discussed in $\S 2$. We also discussed how $\mathrm{CP}$ violation can cause rate asymmetries for both mixing and DCS decays. In this analysis 17. we ignore possible CPV effects which, in the SM, are expected to be small compared to the current experimental sensitivity.

The selection algorithms and analysis cuts are identical for both the mode under study $D^{0} \rightarrow$ $K^{+} \pi^{-}$and the normalizing mode $D^{0} \rightarrow K^{-} \pi^{+}$. To separate these modes we tag the flavour of the neutral $D$ meson via the decay $D^{*+} \rightarrow D^{0} \tilde{\pi}^{+}$. In describing the event selection procedure we shall refer to the events consistent with the (dominant) CF process $D^{*+} \rightarrow \tilde{\pi}^{+}\left(K^{-} \pi^{+}\right)$as right-sign (RS) tagged, and to the events consistent with DCS decay or mixing as wrong-sign (WS) tagged.

\subsection{Event selection}

Candidate events consist of a pair of oppositely charged tracks that form a vertex and have a $K \pi$ invariant mass between 1.7 and $2.1 \mathrm{GeV} / c^{2}$. These $D^{0}$ candidates are used as a seed to search for a suitable production vertex consisting of at least two other charged tracks. The production vertex is required to be isolated from both $D$ candidate daughter tracks, the production vertex is also required to be in target material. The production and decay vertices have to be well separated, and both primary and secondary have to be formed with a good confidence level. To remove background that results from a high-momentum track paring with a random low-momentum track to form a $D$ candidate, we apply a momentumdependent cut is applied that removes highly asymmetrical $K \pi$ pairs.

Doubly misidentified $K^{+} \pi^{-}$pairs from $D^{0}$ decays form a broad peak directly under the $D^{0}$ signal in $K^{+} \pi^{-}$and a narrow peak in the $D^{*}-D$

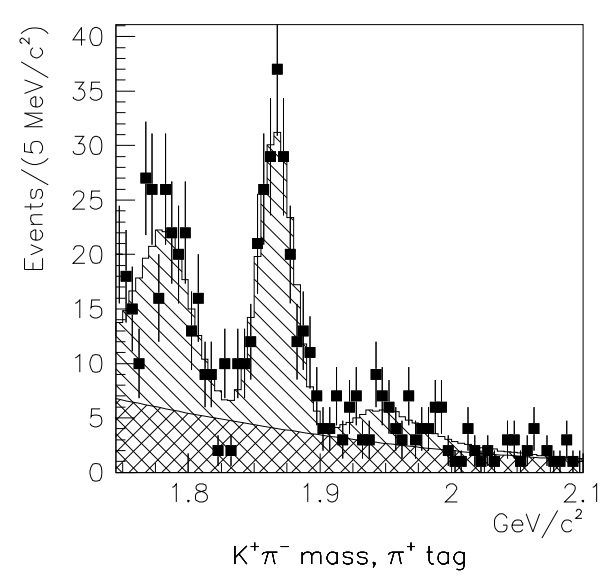

Figure 5. An example fit to the $K \pi$ mass from inside the DCS-like signal region $(146 \mathrm{MeV}<$ $\Delta m<147 \mathrm{MeV})$. Data points are the squares with error bars, fit to data points is shown as the slashed histogram, and the polynomial background fit function is cross-hatched.

mass difference signal region. The mass difference background is indistinguishable from the real WS tagged signal. To eliminate this background, the $K \pi$ invariant mass is computed with the kaon and pion particle hypotheses swapped. Any candidate whose swapped mass is within $\pm 4 \sigma$ of the $D^{0}$ mass is subjected to a cut on the sum of the $K \pi$ separations $\left(W_{\pi}-W_{K}\right)$ for both tracks. Finally, all tracks in the production vertex are tested as potential $\tilde{\pi}$ candidates, and are accepted if within a $\pm 50 \mathrm{MeV} / c^{2}$ window of the nominal $D^{*}-D^{0}$ mass difference, and if they satisfy a loose Cerenkov cut.

\subsection{Fitting technique}

Reflections from partially reconstructed and/or misidentified $D^{0}$ decays with a real $\tilde{\pi}$ can contribute significantly to the WS signal. The measurement method adopted allows one to deal with reflections and feed downs from all known $D^{0}$ decays $\left(D^{0} \rightarrow K^{+} K^{-}\right.$and $D^{0} \rightarrow \pi^{+} \pi^{-}$reconstructed as $D^{0} \rightarrow K^{+} \pi^{-}, D^{0} \rightarrow K^{+} \pi^{-} \pi^{0}$ partially reconstructed, $D^{0} \rightarrow K^{-} \pi^{+} \pi^{0}$ and $D^{0} \rightarrow K^{-} \ell^{+} \nu$ partially reconstructed and doubly 

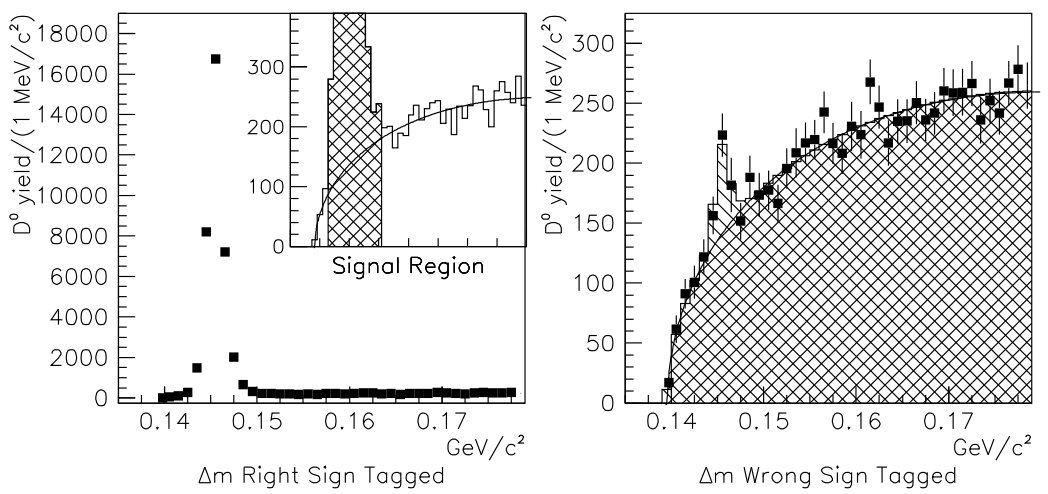

Figure 6. (Left) The RS mass difference distribution. In the inset the signal region used as the WS model is cross-hatched and the solid curve is the fit background shape. (Right) The WS mass difference distribution. Squares with error bars are the fitted $D^{0}$ yields, and histogram is the fit. Background fit is cross-hatched and signal fit is slashed.

misidentified). To deal with these backgrounds we isolate the true $D^{0} \rightarrow K^{+} \pi^{-}$decays by fitting the $K \pi$ invariant mass distribution. The $K \pi$ invariant mass distribution is generated by splitting the RS and WS tagged samples into 1-MeV-wide bands in the $D^{*}-D$ mass differences (Fig.5). Reflections ( $K K$ and $\pi \pi$ ) are fit to montecarlo line shapes, the unstructured background is fit to a polynomial, and the $D^{0}$ signal is fit to a Gaussian. The fitted $D^{0} \rightarrow K \pi$ yields are plotted as a function of the appropriate mass difference bins (Fig.6). By fitting the $D^{0}$ in this way we generate a mass difference distribution that has only true $D^{0} \rightarrow K \pi$ events: the signal is true $D^{0} \rightarrow K \pi$ events with a true $\tilde{\pi}$ tag, and the background is true $D^{0} \rightarrow K \pi$ with a random $\tilde{\pi}$ tag. The random tagged events form a smooth threshold background which is parametrized by the function

$f(\Delta m) \equiv \alpha\left(\Delta m-m_{\pi}\right)^{1 / 2}+\beta\left(\Delta m-m_{\pi}\right)^{3 / 2}(18)$

where $\alpha$ and $\beta$ are free fit parameters. The RS signal is used in the fit as a shape model for the WS signal. At each fit iteration, the RS background - fit outside the signal region - is subtracted from the RS distribution. The final fit parameter is a scale factor used to match the background-subtracted RS signal to the WS signal. In the WS $D^{*}$ signal region the total fit function is the sum of the scaled RS signal and the WS background parametrization. This signal scale factor is the WS to RS branching ratio. The fit is shown in Fig.6, and we obtain a preliminary
Table 3

Recent results on $D^{0} \bar{D}^{0}$ mixing parameters. The CLEO limit on $y^{\prime}$ assumes $x^{\prime}=0$.

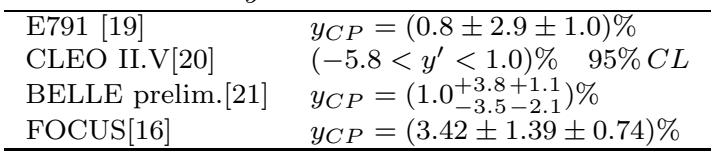

WS to RS branching ratio (statistical error only)

$\frac{\Gamma\left(D^{*+} \rightarrow \pi^{+}\left(K^{+} \pi^{-}\right)\right)}{\Gamma\left(D^{*+} \rightarrow \pi^{+}\left(K^{-} \pi^{+}\right)\right)}=(0.482 \pm 0.093) \%$

From the fit we find $35901 \pm 196$ RS events corresponding to a WS equivalent yield of $173 \pm 34$. The result above is preliminary, only the statistical error is quoted.

\section{CONCLUSIONS}

We have presented a measurement of the $D^{0} \rightarrow$ $K K$ and $D^{0} \rightarrow K \pi$ lifetime indicating that the $K K$ eigenstate has a shorter lifetime than $K \pi$

$y_{C P}=(3.42 \pm 1.39 \pm 0.74) \%$

Our $y_{C P}$ value is compared in Tab. 3 with E791 and BELLE. Also reported is the recent CLEO measurement of $y^{\prime}$ by studying the time evolution of WS hadronic decays (see $\S 2.1$ ). Comparison with the CLEO measurement is not clear because of lack of information on the strong phase $\delta$ [10 11], and any comparison of the $y_{C P}$ and 
the $y^{\prime}$ into one $y$ parameter should be taken cum grano salis. 22.

We have also presented new limits on CPV asymmetries for Cabibbo-suppressed decays such as $K K \pi, K K$ and $\pi \pi$. All results are consistent with zero, with errors at the percent level.

Finally, we showed a preliminary measurement of the WS to RS branching ratio, which, assuming no mixing, corresponds to (statistical error only)

$r_{D C S}=(0.482 \pm 0.093) \%$

This preliminary result preludes a full-blown lifetime analysis which soon will provide the FOCUS measurement of the $r^{\prime}$ mixing parameter.

We wish to acknowledge the assistance of the staffs of the Fermi National Accelerator Laboratory, the INFN of Italy, and the physics departments of the collaborating institutions. This research was supported in part by the U. S. A. National Science Foundation, the U. S. A. Department of Energy, the Italian Istituto Nazionale di Fisica Nucleare and Ministero dell'Università e della Ricerca Scientifica e Tecnologica, the Brazilian Conselho Nacional de Desenvolvimento Científico e Tecnológico, CONACyT-México, the Korean Ministry of Education, and the Korean Science and Engineering Foundation.

\section{REFERENCES}

1. For recent experimental reviews see P. D. Sheldon, hep-ex/9912016; S. Bianco, hep-ex/9911034.

2. I. I. Bigi and A. I. Sanda, "CP violation," Cambridge, UK: Univ. Pr. (2000) 382 p.

3. G. C. Branco, L. Lavoura and J. P. Silva, "CP violation," Oxford, UK: Clarendon (1999) $511 \mathrm{p}$.

4. E. Leader and E. Predazzi, "An introduction to gauge theories and modern particle physics", Cambridge 1996, ISBN $052157742 X$.

5. D.E.Groom et al. (PDG), Eur. Phys. Jour, C15 (2000) 1.

6. I. I. Bigi and N. G. Uraltsev, hep-ph/0005089.

7. T. Liu, CHARM 2000, hep-ph/9408330; FCNC 97, Santa Monica, February 1997 hep$\mathrm{ph} / 9706477$.
8. H.N. Nelson, hep-ex/9908021.

9. I.I. Bigi, 23rd ICHEP, Berkeley, 1986 (SLACPUB-4074), S.C. Loken (Ed.), World Scientific, 1986, p.857 ; G. Blaylock, et al., Phys. Lett. B355, 555 (1995).

10. A. F. Falk, et al., JHEP 9912, 019 (1999).

11. S. Bergmann, et al., Phys. Lett. B486, 418 (2000).

12. F. Buccella et al., Phys.Rev. D51 (1995) 3478.

13. P. L. Frabetti et al. [E687 Coll.], Nucl. Instrum. Meth. A320, 519 (1992).

14. J. M. Link et al. [FOCUS Coll.], Phys. Lett. B491, 232 (2000).

15. P.L. Frabetti et al. [E687 Coll.], Phys. Lett. B370 (1996) 222.

16. J. Wiss [FOCUS Coll.], seminar at Fermilab April 2000; D. Kim, Proc. BEACH2000; J. M. Link et al., Phys. Lett. B485, 62 (2000).

17. J.M. Link [FOCUS Coll.], Proc. DPF2000, Columbus, Ohio (USA), presented by J.M. Link.

18. P.L. Frabetti et al. [E687 Coll.], Phys. Rev. D50 (1994) R2953.

19. E.M. Aitala et al. [E791 Coll.], Phys. Rev. Lett. 83 (1999) 32.

20. R. Godang et al. [CLEO Coll.], Phys. Rev. Lett. 84, 5038 (2000).

21. J. Tanaka, hep-ex/0010078.

22. Papers which have commented on the FOCUS $y_{C P}$ result to-date: $M$. Gronau et al., hep-ph/0010237; A.A. Petrov, hepph/0009160; M. Battaglia, hep-ex/0009026; Y. Nir, hep-ph/0008226; D. Atwood et al., hep-ph/000809d; J.P. Silva, hep-ph/0007214; J.L. Rosner, hep-ph/0007194; J.L. Rosner, hep-ph/0005258; S. Bergmann et al., hep$\mathrm{ph} / 0005181$. 